\section{Custo da alimentação e densidade energética da dieta no Brasil, 2008-2009}

\author{
Cost and energy density of diet in Brazil, \\ 2008-2009
}

\begin{abstract}
This study aimed to evaluate the relationship between the cost and energy density of diet consumed in Brazilian households. Data from the Brazilian Household Budget Survey (POF 2008/2009) were used to identify the main foods and their prices. Similar items were grouped, resulting in a basket of 67 products. Linear programming was applied for the composition of isoenergetic baskets, minimizing the deviation from the average household diet. Restrictions were imposed on the inclusion of items and the energy contribution of the various food groups. A reduction in average cost of diet was applied at intervals of $R \$ 0.15$ to the lowest possible cost. We identified an inverse association between energy density and cost of diet $(p<0.05)$, and at the lowest possible cost we obtained the maximum value of energy density. Restrictions on the diet's cost resulted in the selection of diets with higher energy density, indicating that cost of diet may lead to the adoption of inadequate diets in Brazil.
\end{abstract}

Energy Intake; Food Consumption; Diet
Camila Zancheta Ricardo 1

Rafael Moreira Claro ${ }^{1}$

\section{Introdução}

A obesidade desponta como importante questão de Saúde Pública desde o final do século XX, a princípio atingindo países desenvolvidos, como os Estados Unidos e regiões da Europa 1. Com o passar dos anos, tornou-se uma epidemia de dimensões globais e, atualmente, acomete cerca de 500 milhões de indivíduos adultos em todo o mundo 2 , com os maiores aumentos de prevalência em países em desenvolvimento 1,3 .

No Brasil, o excesso de peso é considerado o problema nutricional mais frequente 4 . O contínuo acréscimo nas taxas de excesso de peso nas últimas décadas resultou em prevalências de excesso de peso e obesidade de respectivamente $49 \%$ e 14,8\% em 2008-2009. Embora esse aumento tenha ocorrido em todos os estratos econômicos, elevações mais intensas foram verificadas na população com menor renda. Enquanto para o conjunto total da população verifica-se aumento de $127,7 \%$ na prevalência de excesso de peso entre 1974-1975 e 2008-2009. Para o estrato da população equivalente ao primeiro quintil da distribuição de renda no país, esse aumento foi de $570,9 \%$ entre os homens e $208,2 \%$ entre as mulheres 4,5 .

As mudanças ocorridas na dieta da população durante esse período certamente contribuíram para o quadro. Nos últimos 35 anos, verificou-se diminuição no consumo de alimentos tradicionais, como arroz e feijão, em paralelo ao 
aumento especialmente no consumo de óleos vegetais, alimentos de origem animal, como carnes, embutidos e leite e derivados, refrigerantes e biscoitos, além de permanência do consumo excessivo de açúcar e insuficiente em frutas e hortaliças, características que revelam um padrão alimentar inadequado, com dietas pobres em alguns micronutrientes e fibras e com alta densidade energética 6,7 .

A densidade energética da dieta, definida como a quantidade de energia fornecida por unidade de peso do alimento ( $\mathrm{kcal} / \mathrm{g}$ ), exerce importante influência no consumo de alimentos e no aporte calórico total da alimentação ${ }^{8}$. Dietas com alta densidade energética tendem a ser mais palatáveis e a aumentar a ingestão de alimentos e energia, o que favorece o aumento excessivo do peso corporal e desenvolvimento de doenças associadas. Estudos realizados em países desenvolvidos encontraram associação inversa entre a densidade energética e o custo da dieta 9,10,11,12,13, confirmando a presença de incentivos econômicos ao consumo de dietas de alta densidade energética. Nesses estudos, todos realizados em mercados com economias desenvolvidas e com cenários de consumo alimentar e preços muito diferentes do brasileiro, a imposição de uma redução ao valor médio da dieta acarretou em elevação de sua densidade energética. Desse modo, famílias de baixa renda ou com orçamento restrito, buscando minimizar seu gasto com alimentos, seriam direcionadas a fazer escolhas alimentares menos saudáveis, de maior densidade energética, no intuito de atingir a necessidade energética mínima dentro das restrições estabelecidas por seu orçamento 14,15. A verificação dessa relação na população brasileira é particularmente importante, uma vez que grande contingente da população ainda possui rendimentos abaixo dos patamares ideais. Contudo, a relação entre custo da dieta e sua densidade energética permanece pouco explorada entre países em desenvolvimento, nos quais a alimentação ainda representa importante parcela do orçamento familiar. O objetivo deste trabalho é avaliar a relação entre o custo da alimentação e sua densidade energética no Brasil.

\section{Metodologia}

\section{Definição da cesta de alimentos}

Foram utilizados dados da Pesquisa de Orçamentos Familiares (POF) realizada entre maio de 2008 e maio de 2009 pelo Instituto Brasileiro de Geografia e Estatística (IBGE). A POF 2008/2009 utilizou plano amostral complexo, por conglo- merados, com sorteio dos setores censitários em um primeiro estágio e de domicílios em um segundo, de modo que, ao final, 55.970 domicílios foram pesquisados. A informação analisada no presente estudo se refere às aquisições de alimentos e bebidas para consumo domiciliar feitas pelas famílias, durante um período de sete dias consecutivos, e registradas diariamente por um dos moradores do domicílio ou por entrevistador do IBGE. O procedimento amostral e as condutas empregadas para coleta de dados são descritos detalhadamente em publicação específica 16 .

Do total de alimentos registrados pela POF ( $\mathrm{N}=1.717$ ), selecionaram-se aqueles com disponibilidade diária média igual ou superior a um grama per capita por dia $(\mathrm{n}=125)$. A cesta de alimentos adotada nas análises, composta por 67 itens, foi obtida após a exclusão dos gêneros isentos de calorias $(n=5)$, das bebidas $(n=12)$ e do agrupamento de 58 produtos semelhantes em 17 itens (Tabela 1).

A fração não comestível da quantidade adquirida dos alimentos foi excluída, empregandose para tal os fatores de correção disponibilizados pelo IBGE 17; e o valor energético (kcal) foi obtido preferencialmente pelo uso da Tabela Brasileira de Composição dos Alimentos 18 e, na ausência de informação sobre determinado alimento nela, foi utilizada a tabela de composição oficial dos Estados Unidos 19.

Os alimentos foram classificados em três macrogrupos de acordo com a extensão e o propósito do processamento ao qual foram submetidos, baseados na classificação proposta por Monteiro et al. 20: (1) alimentos in natura ou minimamente processados (3 grupos e 8 subgrupos); (2) ingredientes para uso culinário ou na indústria de alimentos (3 grupos e 3 subgrupos); e (3) alimentos ultraprocessados (4 grupos e 7 subgrupos). O esquema de agrupamento utilizado é apresentado em detalhes na Tabela 1.

Os preços de cada um dos macrogrupos e grupos de alimentos, em $\mathrm{R} \$ / 1.000 \mathrm{kcal}$, foram obtidos pela média ponderada pelo consumo do total despendido para aquisição dos produtos incluídos em cada um dos grupos.

\section{Densidade energética}

O método utilizado para obter a densidade energética considerou apenas os componentes sólidos da dieta e o leite 21,22 . O cálculo da densidade energética foi realizado dividindo-se o total de energia da dieta (kcal) por seu peso total (g). Para obtenção do peso dos alimentos na forma como esses são habitualmente consumidos (i.e., cozidos, assados, fritos ou grelhados), aplicouse o índice de cocção à quantidade líquida total 
Alimentos com disponibilidade média igual ou superior a um grama per capita/dia nos domicílios brasileiros. Pesquisa de Orçamentos Familiares (POF 2008/2009).

Macrogrupos e grupos de alimentos

Grupo1 (alimentos não processados ou minimamente processados)

Frutas, legumes e verduras

Carnes, ovos e leites

Alimentos básicos I

Grupo 2 (ingredientes para uso culinário ou na indústria de alimentos)

Açúcares

Óleos e gorduras

Alimentos básicos II

Grupo 3 (alimentos ultraprocessados)

Pães, biscoitos e doces

Carnes processadas

Refeições prontas

Outros processados
Subgrupos e alimentos incluídos

Frutas (abacaxi, açaí, banana, goiaba, laranja, limão, maçã, mamão, manga, melancia, melão, tangerina, uva)

Legumes e verduras (abóbora, abobrinha, alface, alho, beterraba, cebola, cenoura, chuchu, pepino, pimentão, repolho, tomate)

Carnes (carne bovina de primeira, carne bovina de segunda, carne suína,

frango/galinha, peixe)

Ovos (ovo de galinha)

Leites (leite desnatado, leite em pó, leite integral, leite semidesnatado)

Cereais (arroz com casca/integral, arroz polido)

Raízes e tubérculos (batata-doce, batata, inhame, mandioca)

Feijões (feijões)

Açúcar (açúcar)

Óleos e gorduras (óleo de soja, creme de leite)

Farináceos e macarrão (farinhas/féculas, macarrão)

Pães (pães)

Biscoitos (biscoito doce/recheado, biscoito salgado)

Doces (chocolate em pó, iogurte de qualquer sabor, leite condensado, sorvete)

Carnes processadas (carne seca, linguiça, mortadela, salsicha)

Refeições prontas (refeição pronta, massa pronta, frango assado ou defumado)

Queijos (queijo minas, queijo muçarela)

Molhos, conservas e gorduras (maionese, margarina vegetal, milho enlatado, molho/massa de tomate) adquirida dos produtos identificada na POF. Os índices de cocção estimados correspondem à divisão entre o valor calórico por $100 \mathrm{~g}$ do alimento cru pelo mesmo valor calórico do alimento cozido (desde que nenhum ingrediente calórico tenha sido adicionado durante o processo de cozimento), tendo sido consultada, para obtenção de tais valores, a tabela de composição de alimentos do U.S. Department of Agriculture (USDA) 19. No caso dos alimentos que usualmente pudessem ser preparados de diversas formas (como cozido ou grelhado), foi utilizado um valor médio entre os índices de cocção definidos para cada forma de preparação (até três formas de preparação por alimento). De forma análoga, para alimentos com consumo possível tanto em sua forma crua quanto cozida foi utilizado um valor médio entre os índices de cocção definidos para forma preparada e o índice de cocção utilizado para consumo na forma de aquisição.

\section{Desenvolvendo dietas com a} programação linear

Programação linear é uma ferramenta matemática útil para guiar decisões quantitativas. Ela se encarrega de encontrar o valor ótimo ou ideal (usualmente por meio de processo de maximização ou minimização) de uma função linear, denominada função objetivo, delimitada por um conjunto de restrições 23 . No presente estudo, a programação linear foi empregada para compor cestas isoenergéticas, compatíveis com a dieta dos brasileiros e valores de gasto definido. $\mathrm{O}$ aporte energético das dietas otimizadas foi fixado em 1.362kcal, disponibilidade de calorias diária per capita encontrada quando utilizada a quantidade média disponível nos domicílios brasileiros dos itens incluídos na cesta de alimentos.

Para assegurar que as dietas fossem o mais próximo possível daquela observada no Brasil, o 
percentil 75 de aquisição dos alimentos foi adotado como limite máximo da quantidade de inclusão de cada item. Foram aplicadas também restrições quanto à contribuição energética de cada um dos grupos e subgrupos de alimentos, que deveria estar entre os percentis 15 e 85 e entre os percentis 25 e 75 de aquisição, respectivamente, considerando-se para tal apenas as famílias que adquiriram esses itens. Os valores reais utilizados como limites podem ser observados na Tabela 2. A função utilizada foi desenvolvida a fim de minimizar o afastamento da dieta média observada nos domicílios brasileiros, ou seja, para identificar, para cada valor de gasto testado, a dieta mais semelhante com a média do país.
Essa função foi definida como a soma dos valores absolutos resultantes da diferença entre a quantidade de cada alimento incluído na dieta e a média de aquisição do alimento entre a população brasileira. Essa função foi adaptada em um modelo linear, conforme metodologia proposta por Darmon et al. 23 .

Aplicando-se as restrições descritas anteriormente, quanto à quantidade de inclusão de cada alimento e contribuição energética dos grupos e subgrupos, chega-se a uma dieta de referência, sobre a qual reduções em seu custo foram aplicadas em intervalos progressivos de $\mathrm{R} \$ 0,15$ até que o menor custo possível, atendendo às restrições impostas, fosse encontrado. O modelo de

Tabela 2

Limites empregados para controle da quantidade de grupos, subgrupos e alimentos incluídos nas cestas.

\begin{tabular}{|c|c|c|}
\hline Grupos & Percentil 15 (kcal) & Percentil 85 (kcal) \\
\hline Frutas, legumes e verduras & 13,18 & 123,31 \\
\hline Carnes, ovos e leites & 46,93 & 389,62 \\
\hline Alimentos básicos I * & 111,78 & 1016,24 \\
\hline Açúcares & 52,88 & 404,66 \\
\hline Óleos e gorduras & 76,44 & 242,88 \\
\hline Alimentos básicos II ** & 30,80 & 274,48 \\
\hline Pães, biscoitos e doces & 93,59 & 297,51 \\
\hline Carnes processadas & 11,88 & 62,46 \\
\hline Refeições prontas & 2,81 & 23,50 \\
\hline Outros processados & 18,75 & 76,04 \\
\hline Subgrupos & Percentil 25 (kcal) & Percentil 75 (kcal) \\
\hline Frutas & 12,48 & 50,41 \\
\hline Legumes e verduras & 5,89 & 12,98 \\
\hline Carnes & 47,06 & 167,84 \\
\hline Ovos & 7,78 & 13,42 \\
\hline Leites & 17,57 & 116,14 \\
\hline Cereais & 126,67 & 339,66 \\
\hline Raízes e tubérculos & 5,99 & 23,91 \\
\hline Feijões & 30,64 & 163,44 \\
\hline Açúcar & 81,80 & 309,98 \\
\hline Óleos e gorduras & 104,86 & 201,38 \\
\hline Farináceos e macarrão & 45,67 & 168,82 \\
\hline Pães & 80,40 & 155,99 \\
\hline Biscoitos & 30,79 & 65,38 \\
\hline Doces & 9,53 & 28,51 \\
\hline Carnes processadas & 17,17 & 49,37 \\
\hline Refeições prontas & 4,75 & 18,64 \\
\hline Queijos & 3,31 & 15,70 \\
\hline Molhos, conservas e gorduras & 21,11 & 43,86 \\
\hline
\end{tabular}

(continua) 
Tabela 2 (continuação)

\begin{tabular}{|c|c|c|}
\hline Alimentos & - & Percentil 75 (g) \\
\hline Abacaxi & - & 5,56 \\
\hline Açaí & - & 0,00 \\
\hline Banana & - & 28,00 \\
\hline Goiaba & - & 1,35 \\
\hline Laranja & - & 19,80 \\
\hline Limão & - & 2,02 \\
\hline Maçã & - & 8,16 \\
\hline Mamão & - & 7,89 \\
\hline Manga & - & 3,62 \\
\hline Melancia & - & 12,98 \\
\hline Melão & - & 1,72 \\
\hline Tangerina & - & 4,17 \\
\hline Uva & - & 3,14 \\
\hline Abóbora & - & 2,92 \\
\hline Abobrinha & - & 1,65 \\
\hline Alface & - & 3,46 \\
\hline Alho & - & 1,74 \\
\hline Beterraba & - & 1,69 \\
\hline Cebola & - & 11,47 \\
\hline Cenoura & - & 5,55 \\
\hline Chuchu & - & 3,12 \\
\hline Pepino & - & 1,82 \\
\hline Pimentão & - & 2,02 \\
\hline Repolho & - & 3,62 \\
\hline Tomate & - & 17,36 \\
\hline Carne bovina de primeira & - & 22,07 \\
\hline Carne bovina de segunda & - & 25,17 \\
\hline Carne suína & - & 1,62 \\
\hline Frango/galinha & - & 44,26 \\
\hline Peixe & - & 1,16 \\
\hline Ovo de galinha & - & 10,38 \\
\hline Leite desnatado & - & 12,08 \\
\hline Leite em pó & - & 3,35 \\
\hline Leite integral & - & 134,77 \\
\hline Leite semidesnatado & - & 1,02 \\
\hline Arroz com casca/integral & - & 1,56 \\
\hline Arroz polido & - & 87,35 \\
\hline Batata-doce & - & 2,13 \\
\hline Batata & - & 21,84 \\
\hline Inhame & - & 0,83 \\
\hline Mandioca & - & 5,56 \\
\hline Feijões & - & 25,99 \\
\hline Açúcar & - & 67,58 \\
\hline Óleo de soja & - & 21,92 \\
\hline Creme de leite & - & 1,49 \\
\hline Farinhas/Féculas & - & 31,44 \\
\hline Macarrão & - & 12,09 \\
\hline Pães & - & 51,18 \\
\hline Biscoito doce/recheado & - & 8,17 \\
\hline Biscoito salgado & - & 6,57 \\
\hline
\end{tabular}

(continua) 
Tabela 2 (continuação)

\begin{tabular}{lcc}
\hline Alimentos & - & Percentil 75 (g) \\
\hline Chocolate em pó & - & 2,68 \\
logurte de qualquer sabor & - & 7,10 \\
Leite condensado & - & 2,59 \\
Sorvete & - & 2,40 \\
Carne seca & - & 1,36 \\
Linguiça & - & 7,85 \\
Mortadela & - & 3,00 \\
Salsicha & - & 4,66 \\
Refeição pronta & - & 2,43 \\
Massa pronta & - & 1,99 \\
Frango assado ou defumado & - & 2,92 \\
Queijo minas & - & 2,67 \\
Queijo muçarela & - & 2,62 \\
Maionese & - & 1,58 \\
Margarina vegetal & - & 5,44 \\
Milho enlatado & - & 4,89 \\
Molho/Massa de tomate & - & 5,12 \\
\hline
\end{tabular}

Nota: os percentis empregados para redução da contribuição energética de grupos e subgrupos de alimentos foram obtidos considerando apenas as famílias que adquiriram os produtos presentes neles.

* Cereais, raízes e tubérculos e feijões;

** Farináceos e macarrão.

programação linear não permite que a soma dos valores absolutos resultantes da diferença entre a quantidade de cada alimento incluído na dieta de referência e a média de aquisição dele entre a população brasileira seja exatamente igual a zero, de modo que pequenas diferenças podem ser observadas entre o consumo médio da população brasileira e a dieta de referência utilizada no estudo. O modelo foi formulado com a utilização do suplemento solver do programa Microsoft Office Excel 2007 (Microsoft Corp., Estados Unidos).

A associação entre a densidade energética da dieta e seu custo foi estudada por meio de teste de correlação de Wilcoxon entre as variáveis, adotando-se $\mathrm{p}<0,05$ como nível de significância.

\section{Resultados}

A Tabela 3 apresenta o valor médio dos macrogrupos e grupos de alimentos além do valor mínimo e máximo de seus componentes. Os maiores preços $(\mathrm{R} \$ / 1.000 \mathrm{kcal})$ foram encontrados para frutas, legumes e verduras $(\mathrm{R} \$ 5,14)$, refeições prontas (R\$5,18), e carnes, ovos e leites ( $R \$ 3,45)$. Já os menores valores foram encontrados para os açúcares $(\mathrm{R} \$ 0,27)$, óleos e gorduras $(\mathrm{R} \$ 0,34) \mathrm{e}$ para os alimentos básicos pertencentes ao grupo $2(\mathrm{R} \$ 0,56)$.
A soma das calorias dos alimentos selecionados foi igual a $1.362 \mathrm{kcal}$, cerca de $85 \%$ do total de energia presente no conjunto completo dos alimentos da POF 2008/2009 7. Quando excluídos da relação de alimentos obtida no inquérito nacional, aqueles produtos isentos de calorias e as bebidas, o preço estimado foi de $\mathrm{R} \$ 1,61 / 1.000 \mathrm{kcal}$ para uma densidade energética de $1,78 \mathrm{kcal} / \mathrm{g}$. Na dieta de referência, os valores encontrados foram semelhantes, respectivamente iguais a $\mathrm{R} \$ 1,67 / 1.000 \mathrm{kcal}(\mathrm{R} \$ 2,28$ para a cesta de alimentos completa) e $1,74 \mathrm{kcal} / \mathrm{g}$.

Identificou-se associação inversa entre a densidade energética e o preço da dieta $(\mathrm{p}<0,05)$. Com a restrição progressiva no custo total da dieta, iniciada a partir do valor da cesta de referência $(\mathrm{R} \$ 2,28)$, verificou-se aumento de sua densidade energética, e, no menor custo possível $(\mathrm{R} \$ 1,10)$, o valor de densidade energética atingiu seu ponto máximo, 2,13kcal/g (Figura 1).

A imposição de restrições sobre preço da cesta de alimentos resultou no aumento na proporção de ingredientes para uso culinário e alguns alimentos tradicionais da dieta brasileira, em detrimento da participação dos demais alimentos. $\mathrm{O}$ aumento da densidade energética deveu-se, especialmente, ao crescimento da participação do açúcar além de cereais e leguminosas em substituição a alimentos in natura e ultraprocessados. A maioria dos gêneros do grupo 1 tiveram 
Tabela 3

Preço (R\$) por 1.000kcal dos grupos de alimentos. Pesquisa de Orçamentos Familiares (POF 2008/2009).

\begin{tabular}{lccc}
\hline Macrogrupos e grupos de alimentos & $\mathbf{n}$ & Preço médio (R\$/1.000 kcal) & Variação de preços (R\$/1.000 kcal) \\
\hline Grupo 1 & 42 & 1,90 & $0,43-49,97$ \\
$\quad$ Frutas, legumes e verduras & 25 & 5,14 & $2,10-49,97$ \\
Carnes, ovos e leites & 10 & 3,45 & $2,27-6,69$ \\
Alimentos básicos I * & 7 & 0,65 & $0,43-2,74$ \\
Grupo 2 & 5 & 0,39 & $0,27-2,32$ \\
Açúcares & 1 & 0,27 & $0,27-0,27$ \\
Óleos e gorduras & 2 & 0,34 & $0,31-2,32$ \\
Alimentos básicos II ** & 2 & 0,56 & $0,46-0,91$ \\
Grupo 3 & 20 & 1,90 & $1,37-10,92$ \\
Pães, biscoitos e doces & 7 & 1,59 & $1,37-8,62$ \\
Carnes processadas & 4 & 2,38 & $1,90-5,30$ \\
Refeições prontas & 3 & 5,18 & $4,72-5,77$ \\
Outros processados & 6 & 2,06 & $0,89-10,92$ \\
Total & 67 & 1,61 & $0,31-10,92$ \\
\hline
\end{tabular}

* Cereais, raízes e tubérculos e feijões;

** Farináceos e macarrão.

Figura 1

Impacto de oito reduções no custo * total da dieta sobre sua densidade energética, estimado por meio de programação linear.

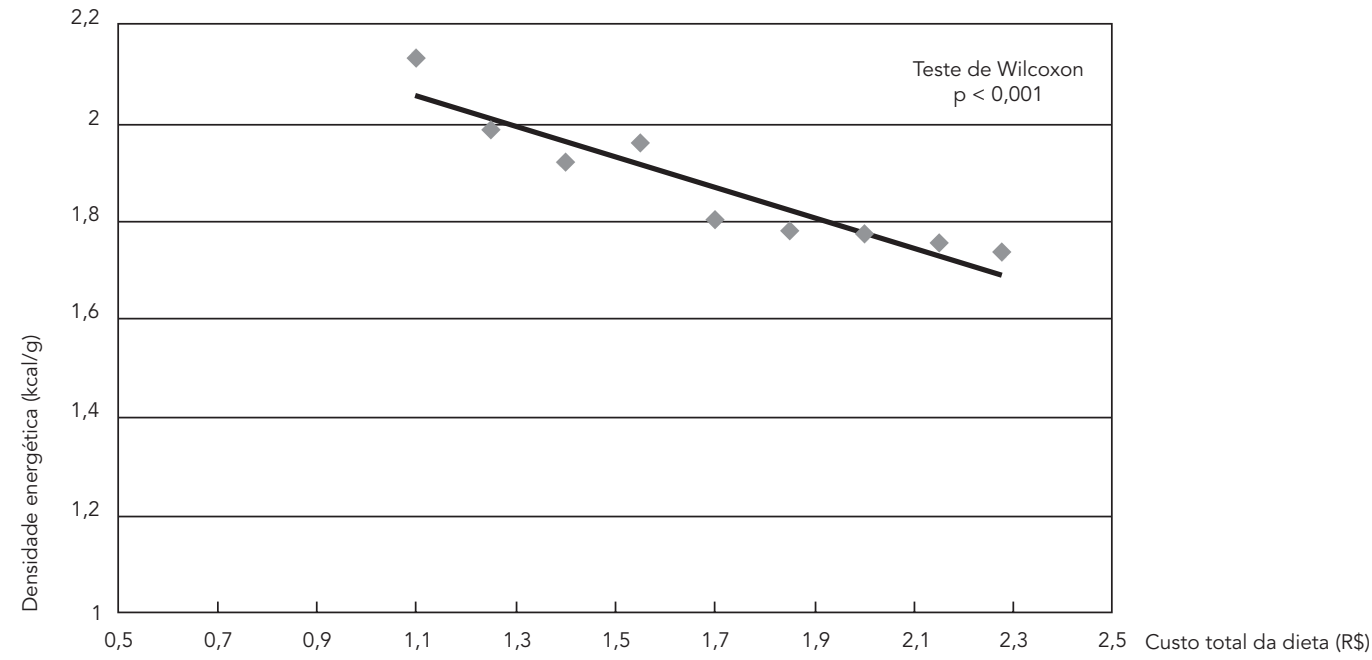

* Reduções progressivas de $\mathrm{R} \$ 0,15$. 
redução acentuada, particularmente os alimentos frescos, representados por frutas, verduras e legumes e carnes, ovos e leites (Figura 2).

\section{Discussão}

Os resultados deste estudo demonstram que a imposição de uma redução no custo médio da dieta está associada ao aumento de sua densidade energética. Observou-se uma elevação progressiva na densidade energética da dieta de referência $(1,74 \mathrm{kcal} / \mathrm{g})$ com a imposição de restrições em seu custo até que ela atingisse seu valor máximo $(2,13 \mathrm{kcal} / \mathrm{g})$ na dieta de menor custo. Tal aumento da densidade energética deveu-se, sobretudo, ao crescimento da participação de ingredientes para uso culinário em substituição a alimentos in natura e ultraprocessados. Destaca-se a redução acentuada no consumo de alimentos de alto valor nutricional e baixa densidade energética, como frutas, verduras e legumes (de 3,8\% para $1,3 \%$ das calorias totais) e carnes, ovos e leites (de $19,8 \%$ para $5,3 \%$ das calorias totais) e o aumento no consumo de alimentos de baixo valor nutricional e alta densidade energética como o açúcar de mesa (de $6,1 \%$ para $19,2 \%$ das calorias to- tais). Esses resultados evidenciam a presença de obstáculos econômicos para a adoção de dietas saudáveis - de baixa densidade energética, com alto teor de frutas, verduras e legumes e outros alimentos in natura - especialmente por famílias de baixa renda.

Vale ressaltar que os resultados apresentados no presente estudo se baseiam em simulações matemáticas e não em observações realizadas nos domicílios brasileiros. Sendo assim, são intimamente dependentes da validade dos critérios adotados na construção do modelo de programação linear utilizado ${ }^{9}$. Um procedimento criterioso foi utilizado para que as cestas de alimentos compostas pelo modelo de programação linear fossem compatíveis com o hábito de aquisição de alimentos observado na população. Os alimentos incluídos foram aqueles mais relevantes (aquisição média $\geq 1 \mathrm{~g} /$ per capita/dia na população brasileira) e restrições foram impostas para que a quantidade de cada alimento e proporção dos grupos e subgrupos não se distanciasse demasiadamente do verificado nos domicílios brasileiros. É importante destacar que tais restrições se baseiam na própria distribuição da aquisição dos alimentos observada para a população brasileira na POF 2008/2009. Outra limitação se refere

Figura 2

Participação (\%) de macrogrupos e grupos de alimentos no total de calorias da cesta de alimentos, de acordo com a redução no custo.

2a) Grupo 1

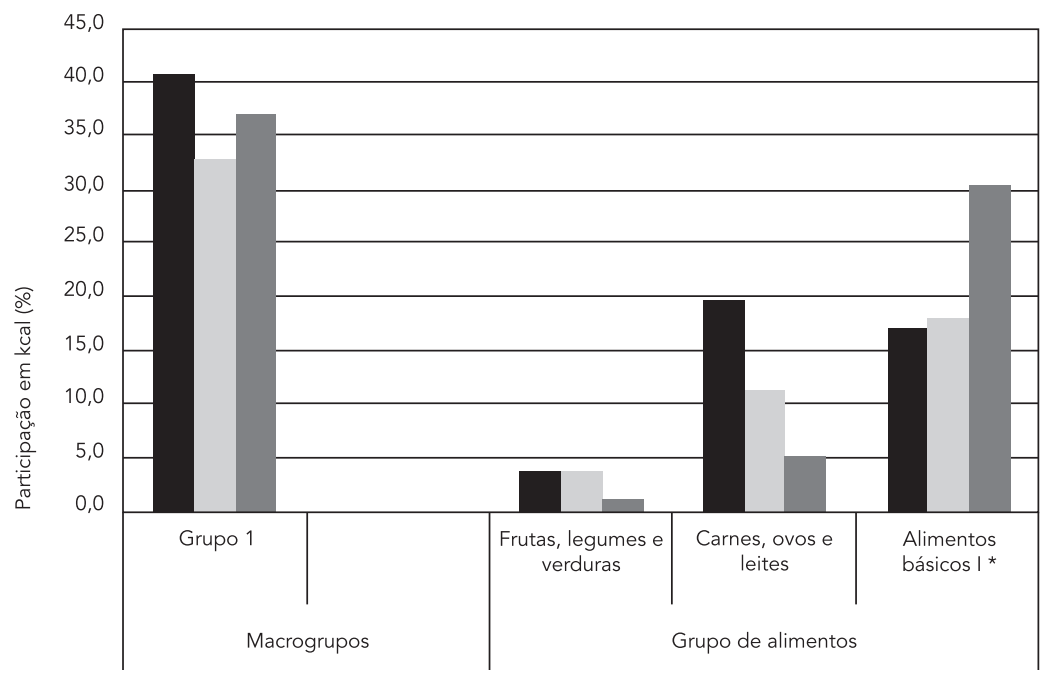

Sem redução de preço

$\prod$ Redução de preço intermediária

Maior redução de preço possíve

(continua) 
2b) Grupo 2
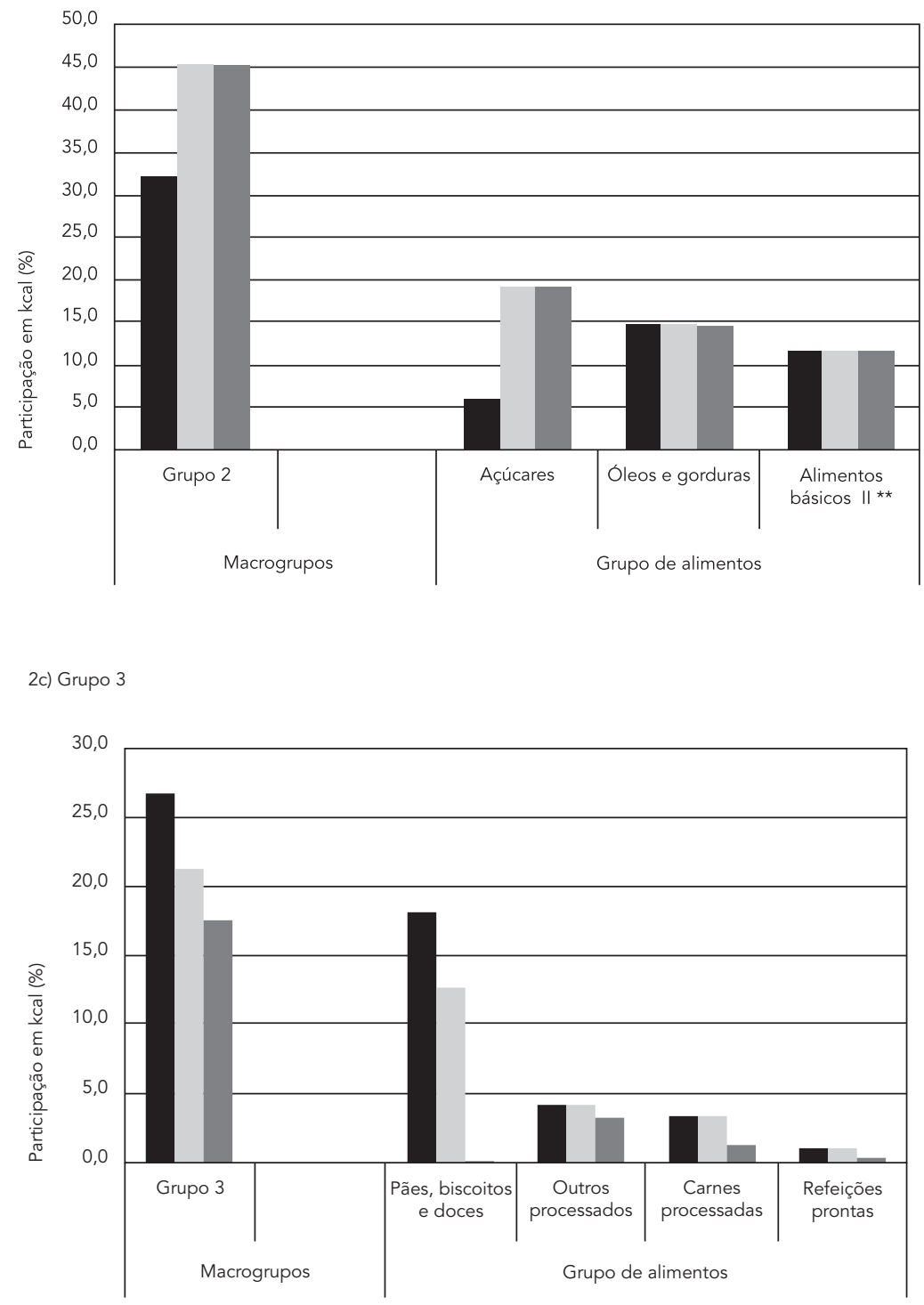

Sem redução de preço $\bigsqcup$ Redução de preço intermediária Maior redução de preço possível

* Cereais, raízes e tubérculos e feijões:

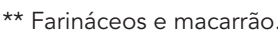

ao fato de que neste estudo tenham sido incluídos apenas os registros de alimentos adquiridos para consumo domiciliar, excluindo-se, portanto, a parcela da alimentação realizada fora de casa. Contudo, no Brasil, a proporção de alimentos adquiridos para alimentação no domicílio é bastante representativa, abrangendo $66,9 \%$ do total gasto com alimentação na área urbana e 82,5\% na área rural do país 16.
Não há consenso na literatura sobre o método mais adequado para o cálculo da densidade energética da dieta $21,22,24$. No presente estudo, optou-se por utilizar o método que inclui todos os alimentos sólidos e o leite 21,22 . Uma vez que o teor de água de um alimento é o maior determinante de sua densidade energética 25 , a inclusão de bebidas no cálculo da densidade energética da dieta seria capaz de influenciar tal estimativa de 
maneira desproporcional 26. Desse modo, optouse pela exclusão das bebidas adoçadas, itens que possuem grande proporção de água adicionada em sua constituição e, consequentemente, baixa densidade energética. Tais produtos, ao contrário dos alimentos sólidos de baixa densidade energética, se relacionam diretamente com o consumo excessivo de alimentos e com o ganho excessivo de peso por serem capazes de prejudicar a capacidade do organismo de reconhecer seu teor calórico, prejudicando a regulação do balanço energético ${ }^{27}$. Além disso, uma vez que tais produtos possuem alto custo por equivalente energético 28 , sua presença nas dietas de menor custo - reduzindo sua densidade energética - seria improvável.

Este é o primeiro estudo dedicado a analisar a relação entre a densidade energética da dieta e seu custo por meio de programação linear envolvendo parâmetros de consumo alimentar referentes à população brasileira. Os resultados são condizentes com os disponíveis na literatura para países desenvolvidos 8,10,13 e indicam que, ainda que o hábito alimentar, o cenário de preço dos alimentos e a cultura sejam bastante distintos, a relação inversa entre densidade energética e custo da dieta está presente também em países em desenvolvimento, o que pode contribuir para o planejamento de estratégias de melhora da qualidade da alimentação nesses países.

Estudo realizado com objetivo e metodologia semelhantes aos adotados neste artigo, baseado em questionário de frequência alimentar de 837 indivíduos adultos e informações da publicação nacional de preço médios dos alimentos na França, encontrou resultados semelhantes, em que a densidade energética, calculada pela exclusão apenas as bebidas alcoólicas e isentas de calorias, passou de 1,45kcal/g e 1,28kcal/g para $2,15 \mathrm{kcal} / \mathrm{g}$ e $1,99 \mathrm{kcal} / \mathrm{g}$, respectivamente, para homens e mulheres, quando o custo foi reduzido aproximadamente à metade do valor médio da dieta 9. Já um trabalho que avaliou a dieta de 112 mulheres de baixa renda da Califórnia, Estados Unidos, também mediante análise de questionário de frequência alimentar, encontrou diferença significante na densidade energética da dieta, tanto quando ela foi obtida considerando apenas os componentes sólidos da dieta, quanto calculada com todos os alimentos e bebidas, exceto água 10. A comparação de nossos achados com esses estudos é dificultada, já que seus resultados foram expostos segundo gênero e a densidade energética estimada de formas distintas quanto à inclusão e exclusão de bebidas, todavia os resultados são unânimes em indicar associação inversa entre o custo da dieta e sua densidade energética. Essa mesma relação foi encontrada ainda noutros estudos observacionais tanto para o conjunto total de alimentos da dieta 8,10 quanto também para itens alimentares analisados isoladamente 12,13,29.

Nas dietas de baixo custo, as quantidades de frutas, verduras e legumes e carnes são diminuídas da dieta enquanto se aumenta a participação de açúcares, grãos e óleos e gorduras, resultando em dietas de alta densidade energética e reduzida qualidade nutricional 23,30 . De fato, em nosso estudo os itens mais baratos foram alimentos de alta densidade energética, como os açúcares (R\$0,27/1.000kcal) e óleos e gorduras $(\mathrm{R} \$ 0,34 / 1.000 \mathrm{kcal})$. O alto consumo desses alimentos possibilitaria a manutenção do aporte energético da dieta para famílias de baixa renda frente a um orçamento restrito 29,31,32. Por outro lado, os altos preços encontrados para as frutas, legumes e verduras $(\mathrm{R} \$ 5,14)$ e para as carnes, ovos e leites $(\mathrm{R} \$ 3,45)$ agiriam como um importante limitante do consumo desses alimentos pela população mais pobre. Do mesmo modo, a participação de todos os subgrupos representantes dos alimentos ultraprocessados, produtos que, de forma geral, apesar de possuírem perfil nutricional adverso, caracterizado por maior densidade energética e maior teor de açúcar adicionado, gordura saturada e sódio e baixa quantidade de fibras 33, possuem preço elevado, também diminuiu nas dietas de menor custo. O custo dos alimentos é dinâmico e pode variar intensamente com o passar do tempo 34. Especialmente no que tange ao mercado nacional, evidências demonstram diminuição do preço dos alimentos ultraprocessados em relação ao conjunto dos demais alimentos da dieta, especialmente frutas, legumes e verduras e carnes frescas, nos últimos trinta anos 34 .

Uma vez que a alta densidade energética da dieta está associada ao consumo excessivo de alimentos 26 , nossos resultados apontam a baixa renda como um fator de risco para o consumo excessivo de alimentos e, consequentemente, para o ganho excessivo de peso e obesidade, tendo em vista a relação inversa entre o custo da dieta e sua densidade energética. Logo, políticas públicas desenvolvidas no intuito de reverter esse cenário devem ser urgentemente consideradas. O aumento da renda das famílias ou a redução do preço dos alimentos de baixa densidade energética em relação aos demais produtos são medidas indicados como possíveis formas de melhorar a qualidade da alimentação da população brasileira. Políticas públicas de transferência de renda são a principal forma de promover elevação da renda de uma parcela da população de forma direta e imediata. Tais políticas já são adotadas no Brasil há dez anos e ainda que elas resultem em 
maior quantidade de alimentos adquiridos, esse aumento é maior para alimentos altamente calóricos e de baixo valor nutritivo 35,36 . As políticas para redução de preço, em princípio, apresentam maior propensão a obter resultados imediatos e efetivos, visto que afetam de forma direta e direcionada a escolha dos consumidores 37 .

A relação inversa entre o custo da dieta e sua densidade energética indica que a melhora da dieta da população brasileira, sobretudo das famílias de menor renda, passa por medidas que sejam capazes tanto de reduzir o preço de alimentos frescos, de baixa densidade energética, quanto elevar o preço dos alimentos de alta densidade energética e baixo valor nutricional.

\section{Resumo}

Este estudo tem por objetivo avaliar a relação entre o custo da alimentação e a densidade energética da dieta consumida nos domicílios brasileiros. Utilizaram-se dados da Pesquisa de Orçamentos Familiares (POF-2008/2009) para identificação dos alimentos com consumo mais relevante e seus preços. Tais alimentos foram agrupados, resultando em uma cesta de 67 produtos. Empregou-se a programação linear para composição de cestas isoenergéticas, minimizando o afastamento da dieta média encontrada nos domicílios. Foram impostos limites para quantidade de inclusão dos itens e contribuição energética dos grupos de alimentos; e uma redução no custo médio da dieta foi aplicada a intervalos de R\$0,15 até o menor custo possível. Identificou-se associação inversa entre densidade energética e preço da dieta $(p<0,05)$, e, no menor custo possível, obteve-se o valor máximo de densidade energética. Verificou-se que restrições no custo da alimentação resultaram na seleção de dietas com maior densidade energética, indicando que o custo da alimentação pode conduzir à adoção de dietas inadequadas no Brasil.

Ingestão de Energia; Consumo de Alimentos; Dieta

\section{Colaboradores}

C. Z. Ricardo participou da revisão da literatura, organização e análise de dados, redação do artigo, construção das tabelas e figuras e aprovou a redação final do artigo. R. M. Claro idealizou o projeto, orientou as etapas de análise dos dados, redação e revisão do texto e aprovou a redação final do artigo.

\section{Agradecimentos}

C. Z. Ricardo recebe bolsa de iniciação científica da Fundação de Amparo à Pesquisa do Estado de São Paulo (FAPESP; processo 2011/13227-8). R. M. Claro recebe bolsa de pós-doutorado da FAPESP (processo 2010/08421-7). 


\section{Referências}

1. Caballero B. The global epidemic of obesity: an overview. Epidemiol Rev 2007; 29:1-5.

2. World Health Organization. Obesity and overweight. http://www.who.int/mediacentre/fact sheets/fs311/en/index.html (acessado em 07/ Mai/2012).

3. Popkin BM, Gordon-Larsen P. The nutrition transition: worldwide obesity dynamics and their determinants. Int J Obes Relat Metab Disord 2004; 28 Suppl 3:S2-9.

4. Instituto Brasileiro de Geografia e Estatística. Pesquisa de Orçamentos Familiares 2008/2009: antropometria e estado nutricional de crianças, adolescentes e adultos no Brasil. Rio de Janeiro: Instituto Brasileiro de Geografia e Estatística; 2010.

5. Secretaria de Atenção à Saúde, Ministério da Saúde. Indicadores de vigilância alimentar e nutricional: Brasil 2006. Brasília: Ministério da Saúde; 2009.

6. Levy-Costa RB, Sichieri R, Pontes NS, Monteiro CA. Disponibilidade domiciliar de alimentos no Brasil: distribuição e evolução (1974-2003). Rev Saúde Pública 2005; 39:530-40.

7. Instituto Brasileiro de Geografia e Estatística. Pesquisa de Orçamentos Familiares 2008/2009: avaliação nutricional da disponibilidade domiciliar de alimentos no Brasil. Rio de Janeiro: Instituto Brasileiro de Geografia e Estatística; 2010.

8. Darmon N, Briend A, Drewnowski A. Energy-dense diets are associated with lower diet costs: a community study of French adults. Public Health Nutr 2003; 7:21-7.

9. Darmon N, Ferguson E, Briend A. Do economic constraints encourage the selection of energy dense diets? Appetite 2003; 41:315-22.

10. Townsend MS, Aaron GJ, Monsivais P, Keim NL, Drewnowski A. Less-energy-dense diets of lowincome women in California are associated with higher energy-adjusted diet costs. Am J Clin Nutr 2009; 89:1220-6.

11. Drewnowski A, Monsivais P, Maillot M, Darmon N. Low-energy-density diets are associated with higher diet quality and higher diet costs in French adults. J Am Diet Assoc 2007; 107:1028-32.

12. Monsivais P, Drewnowski A. Lower-energy-density diets are associated with higher monetary costs per kilocalorie and are consumed by women of higher socioeconomic status. J Am Diet Assoc 2009; 109:814-22.

13. Waterlander WE, Haas WE, van Amstel I, Schuit AJ, Twisk JWR, Visser M, et al. Energy density, energy costs and income: how are they related? Public Health Nutr 2009; 13:1599-608.

14. Darmon N, Drewnowski A. Does social class predict diet quality? Am J Clin Nutr 2008; 87:1107-17.

15. Monsivais P, Aggarwal A, Drewnowski A. Are socioeconomic disparities in diet quality explained by diet cost? J Epidemiol Community Health 2012; 66:530-5.
16. Instituto Brasileiro de Geografia e Estatística. Pesquisa de Orçamentos Familiares 2008/2009: despesas, rendimentos e condições de vida. Rio de Janeiro: Instituto Brasileiro de Geografia e Estatística; 2010.

17. Instituto Brasileiro de Geografia e Estatística. Estudo Nacional da Despesa Familiar: tabela de composição de alimentos. 4a Ed. Rio de Janeiro: Instituto Brasileiro de Geografia e Estatística; 1996.

18. Núcleo de Estudos e Pesquisas em Alimentação, Universidade Estadual de Campinas. Tabela Brasileira de Composição de Alimentos - TACO. Campinas: Núcleo de Estudos e Pesquisas em Alimentação, Universidade Estadual de Campinas; 2004.

19. United States Department of Agriculture. USDA national nutrient database for standard reference. Beltsville: United States Department of Agriculture; 2002.

20. Monteiro CA, Levy RB, Claro RM, Castro IRR, Cannon G. A new classification of foods based on the extent and purpose of food processing. Cad Saúde Pública 2010; 26:2039-49.

21. Cox DN, Mela DJ. Determination of energy density of freely selected diets: methodological issues and implications. Int J Obes 2000; 24:49-54.

22. Ledikwe JH, Blanck HM, Khan LK, Serdula MK, Seymour JD, Tohill BC, et al. Dietary energy density determined by eight calculation methods in a nationally representative United States population. J Nutr 2005; 135:273-8.

23. Darmon N, Ferguson E, Briend A. A cost constraint alone has adverse effects on food selection and nutrient density: an analysis of human diets by linear programming. J Nutr 2002; 132:3764-71.

24. Johnson L, Wilks DC, Lindroos AK, Jebb SA. Reflections from a systematic review of dietary energy density and weight gain: is the inclusion of drinks valid? Obes Rev 2009; 10:681-92.

25. Drewnowski A. Obesity and the food environment: dietary energy density and diet costs. Am J Prev Med 2004; 27(3 Suppl):154-62.

26. Rolls BJ, Drewnowski A, Ledikwe JH. Changing the energy density of the diet as a strategy for weight management. J Am Diet Assoc 2005; 105(5 Suppl 1):S98-103.

27. Monteiro CA, Castro IRR. Por que é necessário regulamentar a publicidade de alimentos. Ciênc Cult (São Paulo) 2009; 61:56-9.

28. Claro RM, Levy RB, Popkin BM, Monteiro CA. Sugar-sweetened beverage taxes in Brazil. Am J Public Health 2012; 102:178-83.

29. Monsivais P, Drewnowski A. The rising cost of low-energy-density foods. J Am Diet Assoc 2007; 107:2071-6.

30. Andrieu E, Darmon N, Drewnowski A. Low-cost diets: more energy, fewer nutrients. Eur J Clin Nutr 2006; 60:434-6. 
31. Drewnowski A, Specter SE. Poverty and obesity: the role of energy density and energy costs. Am J Clin Nutr 2004; 79:6-16.

32. Basiotis PP. Validity of the self-reported food sufficiency status item in the U.S. In: American Council on Consumer Interests 38th Annual Conference [CD-ROM]. Washington DC: U.S. Department of Agriculture; 1992.

33. Monteiro CA, Levy RB, Claro RM, Castro IRR, Cannon $\mathrm{G}$. Increasing consumption of ultra-processed foods and likely impact on human health: evidence from Brazil. Public Health Nutr 2011; 14:5-13.

34. Mondini L, Martins VA, Margarido MA, Bueno CRF, Claro RM, Levy RB. Evolução dos preços de alimentos em São Paulo, Brasil, 1980-2009: considerações sobre o acesso à alimentação saudável. Informações Econômicas 2012; 42:47-55.
35. Castiñeira BR, Nunes LC, Rungo P. Impacto de los programas de transferencia condicionada de renta sobre el estado de salud: el Programa Bolsa Familia de Brasil. Rev Esp Salud Pública 2009; 83:85-97.

36. Lignani JB, Sichieri R, Burlandy L, Salles-Costa R. Changes in food consumption among the Programa Bolsa Família participant families in Brazil. Public Health Nutr 2010; 14:785-92.

37. Claro RM, Carmo HCE, Machado FMS, Monteiro CA. Income, food prices, and participation of fruit and vegetables in the diet. Rev Saúde Pública 2007; 41:557-64.

Recebido em 15/Mai/2012

Versão final reapresentada em 07/Ago/2012

Aprovado em 17/Ago/2012 